\title{
Combination immune checkpoint inhibitor therapy nivolumab and ipilimumab associated with multiple endocrinopathies
}

\author{
Florence Gunawan, Elizabeth George and Adam Roberts
}

\section{Summary}

Immune checkpoint inhibitors are the mainstay of treatment for advanced melanoma, and their use is being increasingly implicated in the development of autoimmune endocrinopathies. We present a case of a 52-year-old man with metastatic melanoma on combination nivolumab and ipilumimab therapy who developed concurrent hypophysitis, type 1 diabetes mellitus (T1DM) and diabetes insipidus. He presented prior to third cycle of combination treatment with a headache, myalgias and fatigue. Biochemistry and MRI pituitary confirmed anterior pituitary dysfunction with a TSH: $0.02 \mathrm{mU} / \mathrm{L}$ $(0.5-5.5 \mathrm{mU} / \mathrm{L}), \mathrm{fT} 4: 5.2 \mathrm{pmol} / \mathrm{L}(11-22 \mathrm{pmol} / \mathrm{L}), \mathrm{fT} 3: 4.0 \mathrm{pmol} / \mathrm{L}(3.2-6.4 \mathrm{pmol} / \mathrm{L})$, cortisol $(12: 00 \mathrm{~h}):<9 \mathrm{nmol} / \mathrm{L}(74-286 \mathrm{nmol} / \mathrm{L})$, FSH: $0.7 \mathrm{IU} / \mathrm{L}$ (1.5-9.7IU/L), LH: <0.1 IU/L (1.8-9.2 IU/L), PRL: $1 \mathrm{mIU} / \mathrm{L}$ (90-400 mIU/L), SHBG: $34 \mathrm{nmol} / \mathrm{L}$ (19-764 nmol/L) and total testosterone: $<0.4 \mathrm{nmol} / \mathrm{L}(9.9-27.8 \mathrm{nmol} / \mathrm{L})$. High-dose dexamethasone $(8 \mathrm{mg})$ was administered followed by hydrocortisone, thyroxine and topical testosterone replacement. Two weeks post administration of the third cycle, he became unwell with lethargy, weight loss and nocturia. Central diabetes insipidus was diagnosed on the basis of symptoms and sodium of $149 \mathrm{mmol} / \mathrm{L}$ (135-145 mmol/L). Desmopressin nasal spray was instituted with symptom resolution and normalization of serum sodium. Three weeks later, he presented again polyuric and polydipsic. His capillary glucose was $20.8 \mathrm{mmol} / \mathrm{L}$ (ketones of $2.4 \mathrm{mmol}$ ), low C-peptide $0.05 \mathrm{nmol} / \mathrm{L}(0.4-1.5 \mathrm{nmol} / \mathrm{L}$ ) and $\mathrm{HbA} 1 \mathrm{c}$ of $7.7 \%$. T1DM was suspected, and he was commenced on an insulin infusion with rapid symptom resolution. Insulin antibodies glutamic acid decarboxylase (GAD), insulin antibody-2 (IA-2) and zinc transporter-8 (ZnT8) were negative. A follow-up MRI pituitary revealed findings consistent with recovering autoimmune hypophysitis. Immunotherapy was discontinued based on the extent of these autoimmune endocrinopathies.

\section{Learning points:}

- The most effective regime for treatment of metastatic melanoma is combination immunotherapy with nivolumab and ipilumimab, and this therapy is associated with a high incidence of autoimmune endocrinopathies.

- Given the high prevalence of immune-related adverse events, the threshold for functional testing should be low.

- Traditional antibody testing may not be reliable to identify early-onset endocrinopathy.

- Routine screening pathways have yet to be adequately validated through clinical trials.

\section{Background}

Immunotherapy has gained popularity as the new novel agent in cancer treatment. Activation of the immune system however has resulted in many autoimmune adverse effects. Our patient had diabetes insipidus, which is a rare complication. To our knowledge, this is also the first case in the literature reporting concurrent hypophysitis, type 1

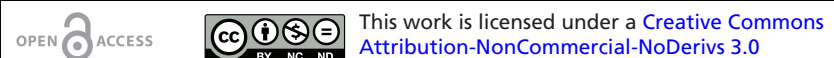
Unported License.
}

(๑) 2018 The authors 
diabetes mellitus and diabetes insipidus in a patient on combination immunotherapy for metastatic melanoma.

\section{Presentation, investigation and initial management}

A 52-year-old firefighter, with stage IV metastatic melanoma was initiated on combination checkpoint inhibitor therapy (dosing regimen: ipilimumab $3 \mathrm{mg} / \mathrm{kg}$ three weekly, nivolumab $1 \mathrm{mg} / \mathrm{kg}$ three weekly) after two melanoma deposits had been resected from his bowel. He had an initial small bowel resection and a follow-up 18FDG PET-CT performed six months later found a mesenteric splanchnic mass with malignant ascites. There was no skin or CNS involvement, and the primary tumor remained unknown. The combination immune checkpoint inhibitor was the primary treatment regimen.

He was not on any regular medications and had been fit and well prior to the discovery of his melanoma. Full blood counts, biochemistry and thyroid function were within normal limits prior to initiation of therapy.

He presented to the emergency department one week following the second cycle of therapy with an abrupt onset of 'the worst ever headache' and transient blurred vision. He was discharged the same day, and his headache resolved over the next week.

Clinical evaluation prior to the third cycle of therapy found him to be hypothyroid with undetectable cortisol. He reported joint aches and low energy and was admitted to hospital for further endocrinologist evaluation. Examination revealed delayed deep tendon reflexes and blood pressure of $112 / 70 \mathrm{mmHg}$, with no evidence of postural hypotension. Heart rate was 80 beats per minute, temperature of $36.2^{\circ} \mathrm{C}$. He had full visual fields; hence, no neuroophthalmology consult or computerized static perimetry was done.

His biochemistry confirmed anterior pituitary dysfunction (TSH: $\quad 0.02 \mathrm{mU} / \mathrm{L} \quad(0.5-5.5 \mathrm{mU} / \mathrm{L}), \quad$ fT4: $5.2 \mathrm{pmol} / \mathrm{L}(11-22 \mathrm{pmol} / \mathrm{L})$, fT3: $4.0 \mathrm{pmol} / \mathrm{L}(3.2-6.4 \mathrm{pmol} / \mathrm{L})$, cortisol (12:00h): <9 nmol/L (74-286nmol/L), FSH: 0.7 IU/L (1.5-9.7 IU/L), LH: <0.1 IU/L (1.8-9.2IU/L), PRL: $1 \mathrm{mIU} / \mathrm{L}$ (90-400 mIU/L), SHBG: $34 \mathrm{nmol} / \mathrm{L}(19-764 \mathrm{nmol} / \mathrm{L})$ and total testosterone: $<0.4 \mathrm{nmol} / \mathrm{L}(9.9-27.8 \mathrm{nmol} / \mathrm{L})$. The free testosterone was not measurable. ACTH and GH were not initially tested. Blood glucose was $5.2 \mathrm{mmol} / \mathrm{L}$ and $\mathrm{Na}$ was $143 \mathrm{mmol} / \mathrm{L}(136-145 \mathrm{mmol} / \mathrm{L})$. Full blood examination (FBE), other electrolytes and liver function tests were normal.

An MRI of the brain (Fig. 1) showed mild diffuse enlargement of the pituitary with contrast enhancement
A

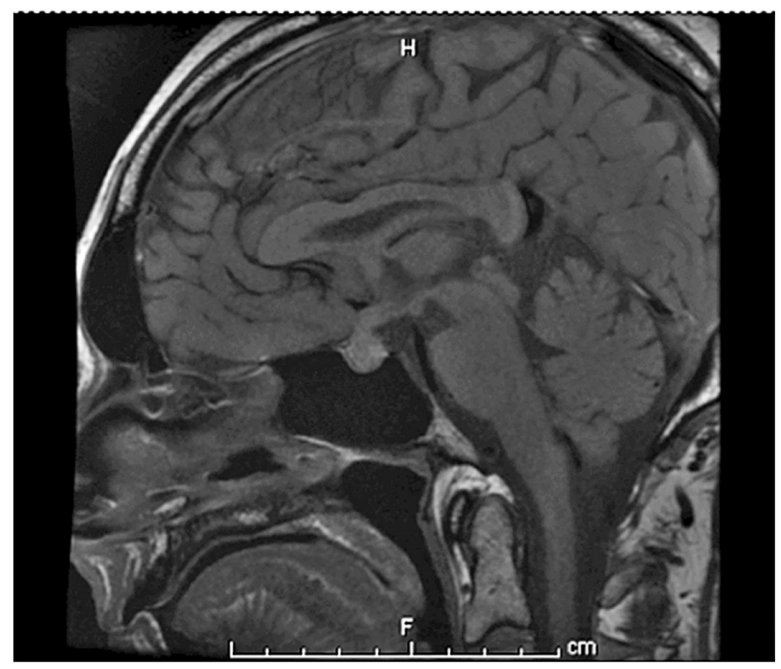

B

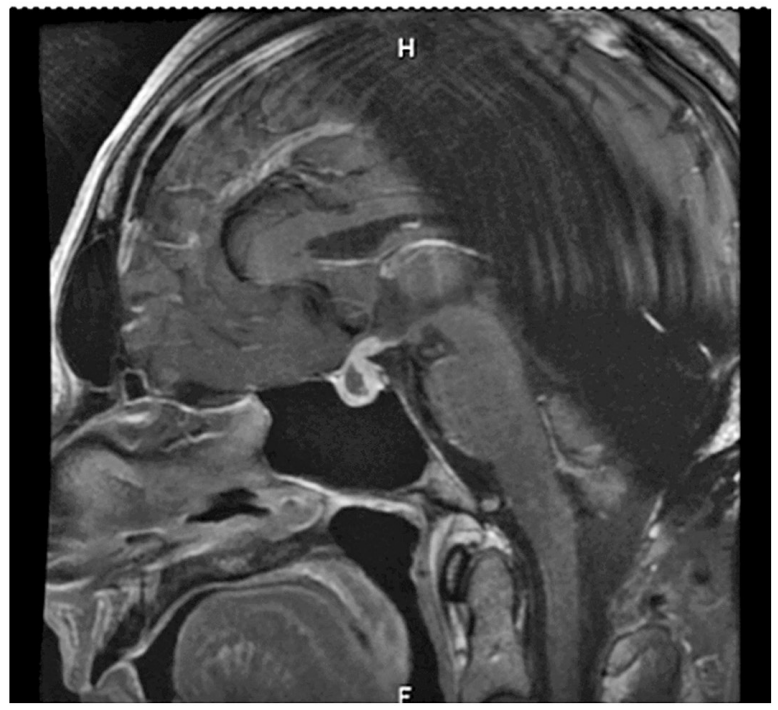

Figure 1

(A) (Pre-contrast): Enlarged pituitary gland. (B) (Post-contrast): Heterogenous anterior pituitary with a cystic component suggestive of possible hemorrhage or hypophysitis.

of the pituitary stalk and posterior pituitary. The anterior pituitary was heterogenous with a cystic component suggestive of a possible hemorrhage. There was no encroachment onto the optic chiasm. The differential diagnosis for this appearance included hypophysitis or metastatic melanoma.

A late attempt to salvage and preserve pituitary function from autoimmune destruction was made by using high-dose IV dexamethasone $8 \mathrm{mg}$ twice daily. Decision was made to switch to oral hydrocortisone 20/10 mg maintenance therapy the next day on the basis of late presentation of the disease and the absence of headache. Thyroxine and topical testosterone were commenced a week later. After some deliberation, the 
third cycle of combination immunotherapy was given two weeks after the onset of hypophysitis on the basis that there was no ongoing toxicity. Decision to continue with the third cycle was based on malignant cells in the peritoneal fluid by the treating oncologist.

Two weeks after recommencement, he became clinically unwell with lethargy, nausea, weight loss and significant polyuria and nocturia. Central diabetes insipidus was diagnosed on the basis of symptoms in the context of pituitary insult and sodium of $149 \mathrm{mmol} / \mathrm{L}$ (135-145 mmol/L). No formal 24-h urine volume quantification, serum or urine osmolality performed at that stage. Desmopressin nasal spray, twice at night, was instituted with relief of symptoms and normalization of his sodium. An attempt at lowering the dose resulted in a return of symptoms and hypernatremia.

Three weeks after his immunotherapy cycle, he presented again polyuric and with polydipsia despite good compliance with his desmopressin. Nausea and vomiting was also a feature. He was admitted into hospital and was found to have a capillary glucose of $20.8 \mathrm{mmol} / \mathrm{L}$ (ketones of $2.4 \mathrm{mmol})$. His C-peptide was $0.05 \mathrm{nmol} / \mathrm{L} \quad(0.4-$ $1.5 \mathrm{nmol} / \mathrm{L}$ ), proinsulin was undetectable at $<3.1 \mathrm{pmol} / \mathrm{L}$ $(<13.3 \mathrm{pmol} / \mathrm{L})$ and $\mathrm{HbA} 1 \mathrm{c}$ was $7.7 \%$. His bicarbonate level was $24 \mathrm{mmol} / \mathrm{L}(22-32 \mathrm{mmol} / \mathrm{L})$. Type 1 diabetes mellitus was suspected, and he was commenced on an insulin infusion for glucose stabilization. The nausea resolved quickly. Insulin antibodies including glutamic acid decarboxylase (GAD), insulin antibody-2 (IA-2) and zinc transporter-8 (ZnT8) were negative. He continued to require a relatively high dose of insulin in the form of a basal bolus regimen of Lantus 22 units daily with NovoRapid three times a day with meals, dosed as per carbohydrate intake with insulin-to-carbohydrate ratio (ICR) of 1:7.5 and insulin sensitivity factor (ISF) of 1:2.4. His weight was $89 \mathrm{~kg}$.

\section{Outcome and follow-up}

A follow-up gadolinium-enhanced MRI of the pituitary (Fig. 2) revealed resolution of the pituitary swelling. The anterior pituitary exhibited no post-contrast enhancement while the posterior pituitary enhanced normally. The findings were considered consistent with recovering autoimmune hypophysitis. He was continued on maintenance dose of hydrocortisone $20 / 10 \mathrm{mg}$ daily, thyroxine, testosterone replacement, desmopressin nasal spray and insulin.

Immunotherapy was discontinued based on the extent of these toxicities. Immunotherapy was discontinued
A

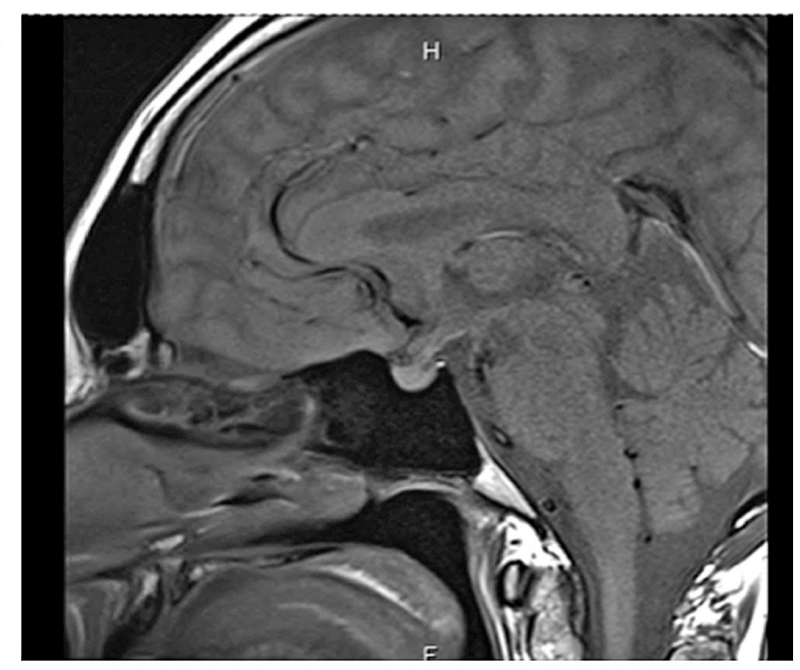

B

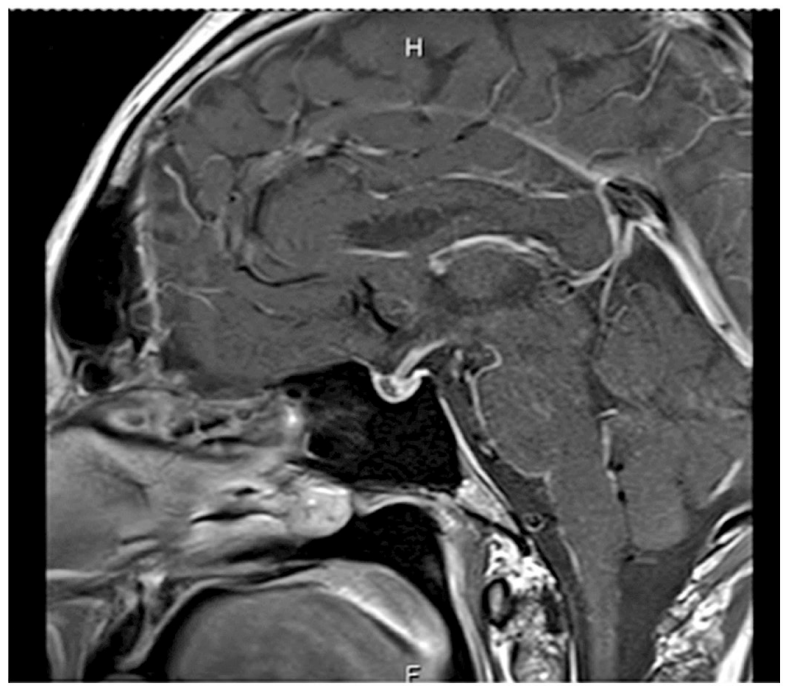

Figure 2

(A) (Pre-contrast): Pituitary swelling has resolved. (B) (Post-contrast): Loss of enhancement of anterior pituitary.

based on the extent of toxicities. The patient remains on insulin, desmopressin and hormone replacement.

\section{Discussion}

Immune checkpoint inhibitor therapy has outperformed targeted molecular therapies in the treatment of advanced melanoma. Cytotoxic T-lymphocyte-associated antigen-4 (CTLA-4) is a protein receptor expressed on T-cells that downregulates the immune system. Ipilimumab is a monoclonal antibody that upregulates anti-tumor immunity by blocking CTLA-4 receptors allowing antigen-presenting cells to recognize tumor cells as targets. Ipilimumab was the first agent to be associated with improvement in overall survival in patients with metastatic melanoma (1). 
The binding of programmed cell death-1 (PD-1) on T-cells to PD-1L on cancer cells also downregulates the immune system. Nivolumab is a monoclonal antibody against the PD1 receptor, which functions to inhibit the downregulation of the immune system toward tumor cells.

The inherent risk of these therapies is autoimmune disease from unrestrained T cell activation. Autoimmune endocrinopathies such as thyroid dysregulation, hypophysitis, adrenal insufficiency and T1DM have been described in the literature. In ipilimumab-treated metastatic melanoma, the incidence of hypophysitis varies from 5 to $17 \%$ with a time to onset between 2 and 4 months, however, more delayed presentations may occur $(2,3,4,5)$. The incidence of hypophysitis with nivolumab monotherapy is lower at $<1 \%$, (compared to ipilimumab) and the median time to onset is 4.9 months (6). Central hypothyroidism is perhaps the most common but multiple anterior pituitary hormone deficiencies do occur (5). Diabetes insipidus on the other hand is rare $(4,5)$. Inflammatory process causing pituitary destruction commonly results in anterior pituitary dysfunction, whereas posterior pituitary function often remains normal. The cause for this is unclear. We postulate that the structural difference of the anterior and posterior pituitary gland could potentially account for this. Anterior pituitary gland is connected to the hypothalamus through a vascular hypothalamushypophyseal portal system. It also synthesizes and secretes hormones. The posterior pituitary gland is made of nerve bundle as an extension of the hypothalamus and it only stores and secretes but does not synthesize hormones. We think that the vascularity of the anterior pituitary gland would potentially predispose it to systemic therapy and its associated toxicities, rendering it more susceptible to damage, compared to the posterior pituitary gland. This theory however needs to be looked into with further studies.

Type 1 diabetes mellitus has been associated with nivolumab (incidence of $0.9 \%$; median time to onset 4.4 months). Patients often present with rapid-onset hyperglycemic ketosis with relatively low $\mathrm{HbA1c}$ and C-peptide levels suggestive of fulminant type 1 diabetes $(7,8)$. Insulin antibodies (GAD, islet cell and IA-2) can be negative, however, genetic testing in antibody-negative patients demonstrates HLA class II DR4 and DQ haplotypes $(7,8)$. The pathophysiology of anti-PD1-induced diabetes is different from the conventional autoimmune antibody positive type 1 diabetes. Glucocorticoid could have contributed to hyperglycaemia in this case; however, it is less likely to be the etiology of diabetes given the low proinsulin and C-peptide level.

Combination therapy has been shown to significantly increase progression-free survival than treatment with ipilimumab alone (9). Immune-related side effects are compounded in combination therapy. The incidence of type 1 diabetes doubles to $1.5 \%$ and the median time to onset is faster at 2.2 months.

Hypophysitis occurs much more commonly in patients treated with combination immunotherapies than either therapy alone. Studies demonstrate an incidence of $9-12 \%$ in combination therapy, compared to $<1 \%$ in nivolumab monotherapy and $7 \%$ in ipilimumab monotherapy. The incidence of hypophysitis further increases with the number of doses of ipilumimab or nivolumab in the combination therapy (10). The median time to onset of hypophysitis is also faster in combination therapy at 2.7 months. A variable number of patients with hypophysitis recover some anterior pituitary function; however, the time to recovery is unpredictable. The rate of discontinuation of therapy due to adverse effects was also higher in combination therapy $(9,10)$.

Declaration of interest

The authors declare that there is no conflict of interest that could be perceived as prejudicing the impartiality of the research reported.

\section{Funding}

This research did not receive any specific grant from any funding agency in the public, commercial or not-for-profit sector.

\section{Patient consent}

Informed consent has been obtained from the patient for publication of the submitted article (file attached).

\section{Author contribution statement}

Florence Gunawan: Consolidated information including investigations results and treatment; writing up case report. Elizabeth George: Writing up case report. Adam Roberts: Treating physician; writing and supervising case report.

\section{References}

1 Hodi FS, O'day SJ, McDermott DF, Weber RW, Sosman JA, Haanen JB, Gonzalez R, Robert C, Schadendorf D, Hassel JC, et al. Improved survival with ipilimumab in patients with metastatic melanoma. New England Journal of Medicine 2010363 711-723. (https://doi. org/10.1056/NEJMoa1003466)

2 Horvat TZ, Adel NG, Dang TO, Momtaz P, Postow MA, Callahan MK, Carvajal RD, Dickson MA, D'Angelo SP, Woo KM, et al. Immune-related 
adverse events, need for systemic immunosuppression, and effects on survival and time to treatment failure in patients with melanoma treated with ipilimumab at Memorial Sloan Kettering Cancer Centre. Journal of Clinical Oncology 201533 3193-3198. (https://doi. org/10.1200/JCO.2015.60.8448)

3 Ryder M, Callahan M, Postow MA, Wolchok J \& Fagin JA. Endocrinerelated adverse events following Ipilumumab in patients with advanced melanoma: a comprehensive retrospective review from a single institution. Endocrine-Related Cancer 201421 371-381. (https://doi.org/10.1530/ERC-13-0499)

4 Faje AT, Sullivan R, Lawrence D, Tritos NA, Fadden R, Klibanski A \& Nachtigall L. Ipilimumab-induced hypophysitis: a detailed longitudinal analysis in a large cohort of patients with metastatic melanoma. Journal of Clinical Endocrinology and Metabolism 201499 4078-4085. (https://doi.org/10.1210/jc.2014-2306)

5 Faje A. Immunotherapy and hypophysitis: clinical presentation, treatment, and biological insights. Pituitary 201619 82-92. (https:// doi.org/10.1007/s11102-015-0671-4)

6 Topalian SL, Hodi FS, Brahmer JR, Gettinger SN, Smith DC, McDermott DF, Powderly JD, Carvajal RD, Sosman JA, Atkins MB, et al. Safety, activity and immune correlates of anti-PD1 antibody in cancer. New England Journal of Medicine 2012366 2443-2454. (https://doi.org/10.1056/NEJMoa1200690)

7 Okamoto M, Okamoto M, Gotoh K, Masaki T, Ozeki Y, Ando H, Anai M, Sato A, Yoshida Y, Ueda S, et al. Fulminant type 1 diabetes mellitus with anti-programmed cell death-1 therapy. Journal of Diabetes Investigation 20167 915-918. (https://doi.org/10.1111/ jdi.12531)

8 Hughes J, Vudattu N, Sznol M, Gettinger S, Kluger H, Lupsa B \& Herold KC. Precipitation of autoimmune diabetes with anti-PD-1 immunotherapy. Diabetes Care 20154 e55-e57. (https://doi. org/10.2337/dc14-2349)

9 Larkin J, Chiarion-Sineli V, Gonzales R, Grob JJ, Cowey CL, Lao CD, Schadendorf D, Dummer R, Smylie M, Rutkowski P, et al. Combined nivolumab and ipilimumab or monotherapy in untreated melanoma. New England Journal of Medicine 2015373 23-24. (https:// doi.org/10.1056/NEJMoa1504030)

10 Wolchok JD, Kluger H, Callahan MK, Postow MA, Rizvi NA, Lesokhin AM, Segal NH, Ariyan CE, Gordon RA, Reed K, et al. Nivolumab plus ipilimumab in advanced melanoma. New England Journal of Medicine 2013369 122-133. (https://doi.org/10.1056/ NEJMoa1302369)

Received in final form 20 December 2017 Accepted 16 January 2018 